\title{
A New World for Better Health
}

\author{
William Edward Hammond ${ }^{1,2}$ \\ ${ }^{1}$ Duke Center for Health Informatics, Duke Medicine, Durham, North Carolina, USA \\ ${ }^{2}$ Clinical \& Translational Science Institute, Duke Medicine, Durham, North Carolina, USA
}

\begin{abstract}
Issue: The healthcare industry is undergoing an exponential change. Our attempts to deal with these changes are inadequate, unimaginative, untimely, and too narrowly focused.
\end{abstract}

Goal: The goal of this paper is to increase the awareness of the technical community - particularly the standard developers and the implementers - about what is happening.

Methods: The paper identifies a number of changes that are occurring and discusses consequences that may result from these changes. Many of these potential changes are recognized through observations relating to new and developing technologies, through the new roles for the set of involved people, and for recognized problems with current systems. The major funded research initiatives were used to predict changes as were the directions a number of industry giants are moving, such as, Apple, Google, IBM, and a number of start-ups. Certainly the hype

\section{Correspondence to:}

William Edward Hammond, PhD, FACMI, FAIMBE, FIMIA, FHL7

Duke Center for Health Informatics, NC 27705, USA

Tel: 919.668.4647

E-mail:william.hammond@duke.edu for Artificial Intelligence, Machine Learning, Deep Learning, and Cognitive Computing is moving toward reality.

Conclusions: Pick your time frame - from 2020 to 2050 - these changes are very likely to occur. Healthcare, as we know it, will change. Computers and robots will replace humans for many of the tasks now done by humans. Population Health will engage all sectors of the world around us - individuals, families, neighborhoods, communities, regions, and countries. Schools, churches, and social groups will become important in health and well-being. Patients will play a major role in managing their own health and healthcare. Becoming over age 60 will not mean waiting to die. New types of data other than just clinical will be just as important as clinical data. Genetic data will be available about everyone and will be used to determine risks.

\section{Keywords}

Healthcare paradigm changes; Big data; Interoperability; Standards; Population health

\section{EJBI 2017; 13(1):3-8}

received: July 14, 2017

accepted: August 30, 2017

published: October 10, 2017

\section{Introduction}

The world we live in today changes at an unbelievable pace. Change is the constant in our world today. Over the next few years, health and healthcare will change in almost every aspect. The advent of mobile devices, wearable sensors, patient reported data, augmented reality, virtual home visits, machine learning, artificial intelligence, data sharing, geospatial coding, and aspects of Big Data will totally change the way, the place, and the how of medical care. Roles of healthcare providers - doctors and nurses - will change. The way we collect data will change. The types of data - not just clinical but social, economic, environmental, behavioral, and genetic data - will change how decisions are made and treatments are determined. Aggregation of data not only for individuals but for regions, states and provinces, countries, and globally will change and produce new problems.
Initiatives in the United States (US) include Population Health, Precision Medicine, Big Data to Knowledge, Health Information Exchanges, Health Analytics and others reflecting these changes. Translational medicine will increase the use of data for multiple purposes [1].

The amount of data collected per person will be in the petabyte range. Input of new data will trigger thousands of clinical decision making algorithms. The required speed of access and navigation to specific data, for speed in calculations, and for effectively presenting decisions and results will require new architectural infrastructures and new concepts in databases. As a consequence of these changes in health and healthcare, the increase in life expectancy at birth has increased over my lifetime by almost 17 years. At my current age, my expected remaining years of life continues to rise from its current prediction of 8.1 years. Technology has 
contributed a significant percentage to that increase. This longer lifespan has raised significant issues in health and healthcare affecting cost, social issues, crowding, and really just what to do with old people. People retiring at the age of 65 now expect to have, on average, an additional 20 years of life. Hence the problem. Factors impacting health include multiple chronic diseases, outliving retirement income, disruptive families, boredom, and overcrowding [2].

These new directions have introduced many new "words" and new ways of thinking about the future. Interoperability and standards that will enable interoperability, global standardization of semantics, cloud computing, integrated care plans, blockchain, quality, provenance, privacy, consumer engagement, socializing, and disruptive innovation are a necessity for success. Modifiers to health now include digital, tele, and virtual.

This paper discusses some of those changes and addresses how HL7 International and the expanded Informatics community needs to address these changes. First, from my observation point, we seem to spend most of our time in working around a problem rather than addressing the problem directly. Second, we seem to address most problems with solutions that are already out of date. Third, we address each problem as a single, isolated problem rather than dealing with the problem in a broader setting. Fourth, when we start to solve a problem, we start with what we know and have available, rather than looking at the best solution then looking to see what exists to solve the problem. Fifth, we ignore the hard problems. Six, they frequently say, "You can't do that because ..." or "That is impossible". We need to design systems for tomorrow and not systems for yesterday.

This paper will discuss a number of factors that are consequences of the changing world of health care. The most significant changes are a result of advances in technology. Those changes are reflected in the continued validation of Moore's Law. Computers are faster, smaller, and more powerful than ever. Costs are way down. The advent of smart phones has had one of the biggest impacts on society since the invention of cars and airplanes. Now, mobile devices and wearable sensors have changed our views of data - how we collect it, and how we use it. Real time, global communications have introduced problems in dealing with world-wide epidemics.

\section{A Changing World}

\subsection{Change from Sick Care to Health}

One of the most significant changes in the health care system is the reimbursement focus change of fee-for-service to accountable care. With fee for service, healthcare institutions attracted the sickest patients because these resulted in the highest revenues. With accountable care organization, the healthiest patients result in the most profit, and sick patients cost money. Hospitals dominated the health scene. Now, we can view hospitalization as a failure of the system.

\subsection{Shift from Provider Focus to Patient Focus}

For much of my life, patients should be seen and not heard. Patients were not allowed to see their data because they would not understand. Further, for the most part, patients were not interested in seeing their data. With the increased interest in a healthy life, with marketing for a healthy life style, with social media and internet searches, patients have become much more sophisticated. Patients do understand to a greater extent their diseases and want to have a major role in their treatment. Personal Health Records and Patient Portals now provide access to their EHRs. I recognized that the data contained in my system EHR are mostly baseline data resulting from my annual check-ups. What happens to me in everyday life - in my working and playing environments are much more reflective of my health state. That data is what I want to share with my provider. This push of data into the healthcare process has taken advantage of technology with mobile devices and wearable sensors that provide significant health status data. Patient Reported Outcomes (PRO) provide critical information about the effectiveness of treatment or a dynamic health status.

\subsection{Restricted Access to Shared Data}

Largely for financial reasons, access to clinical data has been carefully constrained, protected, restricted and controlled. Health care systems compete, and they protect their client base, clinical outcomes, and other sensitive data. The sharing of personalized data across multiple sites causes new challenges in security and privacy [3]. Physicians treat any data from external sources as being of poor quality and of little value. The only data they trust for decision making is the data for which they are responsible. Systems, standards, knowledge, even terminologies and tools developed by vendors and institutions have perceived value and are shared only for a price. One of the consequences is that each group - at all levels of definition - develop what they need independently. In the U.S., each state has responsibility for the development of systems which they do redundantly and slightly differently. However, patients cross state boundaries to receive their care with a high probability that data would not be shared or used across state lines.

Fortunately, we are moving into a period in which the value of sharing data, knowledge, tools, and systems for little or no cost is being accepted. In the U.S., proposals for research grant funding require a section stating how data will be shared with others. Sharing data is a necessity for creating an aggregated Patient-Centric EHR. Big Data and its potential for new knowledge and more data for clinical research is a product of sharing. Rare diseases are less rare as a result of sharing. Pragmatic clinical trials have the potential of being a primary method of clinical research. However, if the EHR is the data source for clinical trials, the demands for quality, completeness and consistency is paramount. These requirements become a mandate for interoperability. Unfortunately, we are not yet there. 


\subsection{New Types of Data}

In 2002 McGinnis and others published a paper in Health Affairs [4] in which they concluded that shortfalls in medical care contributed only $10 \%$ to the health status of an individual. Behavior patterns are responsible for $40 \%$ of the health index, yet we spend only about $5 \%$ of healthcare expenditures at a population level to improve health-related behavior. Fortunately, that amount of money in the new world has increased considerably. Genetic predispositions contribute $30 \%$, social circumstances $15 \%$, and environmental exposures $5 \%$. These findings have not only influenced our healthcare expenditures but are influencing the kinds of data we collect. Current research efforts are directed toward how to collect and use these kinds of data in healthcare. What genetic data should be included in the EHR, and how will it be stored? How should we present socioeconomic and environment data to the clinician? Geospatial coding of data is now becoming part of the EHR. Suddenly, the clinician is faced with thousands of data items in the decision making process. Humans find it difficult to use more than 5-7 facts in decision making. Do physicians now have to become geneticists to effectively understand and use genetic data?

\subsection{Competitive to Collaborative}

Competition drives much of our activities. New economic models are pushing more and more healthcare organizations to create collaborative groups for survival. High-speed transport makes it easier for patients to travel to more distant health care facilities. The increase in cost of overhead such as EHR systems, costly diagnostic equipment, and shortage of trained professionals are forcing small and rural institutions to become part of a larger network. Centers of excellence for specific diseases attract patients to well-known institutions such as Mayo, Cleveland Clinic, Johns Hopkins, Duke, and others. Experts have suggested that healthcare in the future may be delivered by less than 10 healthcare systems in the U.S.

New appreciation for the value of Big Data has encouraged collaborative networks for research. Big Data has the potential to help us to better manage chronic diseases. Federal funding for such collaborative research efforts as the NIH Collaboratory [5] grants and the PatientCentered Outcomes Research Network (PCORnet) [6] are creating access to over 122 million patients. PCORnet is a large network of networks that harnesses the power of collaboration. This network covers much of the U.S.

\subsection{Licensed for Fee to Free and Open Source}

The balance between proprietary systems and open source systems has been constantly evolving. Products, including standards, terminologies, decision support algorithms, tools, rules, and even data that have value to the entire global community are being made available to all at no cost. If the goal is to have everybody use the same systems, then making that product freely available to all makes sense.
From its beginning in 1987, HL7 required membership in HL7 to access its standards. In September 2012, HL7 International standards became licensed at no cost. Across the industry, there is still an inconsistency in what products are available without cost and what require payment. The problem is that nothing is free. There is a cost in creating, distributing, educating, and administrative infrastructure to support the product. We need to develop a business model that will support and fund the open source process.

\subsection{Site Specific to Mobile}

The current model for a person to go to the healthcare facility for both health care and illness care is changing. Getting an appointment in a large medical center, especially an academic medical center, one is faced with long wait times - on the order of months. Urgent Care Centers scattered throughout the region have been created to address this problem. In addition, Walgreens, CVS and others now offer urgent care. Patient satisfaction is further strained by long waits well beyond the scheduled times for the appointments. The cost of care is increased by parking fees that are not insignificant.

With the advent of mobile devices, new models for the delivery of care are appearing. The computational capability, the resolution of the display, and the quality of the incorporated video/camera has offered new methods of communication between individuals. The old house call, long disappeared, is coming back in the form of a smart phone encounter. The time, cost, and inconvenience of traveling to a clinic now is being replaced by a call. Further, the smart phone can be combined with a smart application programming interface (API) to collect data, to provide a reminder, to educate the patient, to answer a question, or a number of other tasks.

Wearable sensors can now monitor about 20 data elements in addition to patient reported data. The challenge to the IT community is how to integrate this data and how to validate its accuracy. Apple [7], for example, is addressing health as part of its business model. Apple introduced the Apple Watch which tracks activity and heart rate. Development continues to incorporate more sensing devices to measure and track other important data items. It is interesting to note that that just the reporting feature of step activity has motivated persons to set and reach exercise goals. Apple's Health app on IOS and the HealthKit framework support the aggregation of health-related data. ResearchKit is an open source framework that allows researchers and developers to create apps for medical research. At 6 months, over 100,000 people were using medical apps, and that number is rapidly growing. An example of these apps include autism (Duke), melanoma-screening (Oregon Health \& Science University), and EpiWatch, a seizure-tracking app. CareKit allows individuals to manage their own medical conditions. Other groups, including Fitbit, are developing sensor functionality.

The challenge for the IT community is how to integrate these devices into the EHR and into the healthcare process. It is likely that apps will be developed independently. We need to develop methods 
to integrate and aggregate the different data items into a cohesive whole. For example, combining activity measurements with heart rate and blood pressure measurements will provide much more information than either measurement alone. Adding one's calendar events would add additional information. The potential of these individual real-time measurements will significantly impact the health status of individuals. Heart attacks and strokes could be detected before they happen, and measures taken to prevent or minimize the event before it happens. These disruptive innovations will impact the sites of care, the delivery of care, communication, and interventions.

\subsection{The Aging Population}

The aging of the population is one of the most significant trends of the 21 st century [8]. One in eight people (12.5\%) in the world are age 60 or over. As long as fertility rates continue to decline and life expectancy continues to rise, older people will steadily increase as a proportion of the population. By 2050, the percentage of persons over 60 increase to $22 \%$. The problem for society is what to do with the aged population and how can we afford to support this group. Population Health addresses this problem with incentives including appropriate use of this population and independent aging in the home. Many of the technological developments already discussed provide solutions to many of these problems. Sensors, videos with real time analysis, medication assists, and fall-proofing the homes are examples. The use of robotics in the home have the potential of providing acceptable communications with humans. Real-time data with real-time analytics can predict impending healthnegative events. Prearranged connections to a healthcare facility will provide direct triaging and timely interventions.

\section{What these Changes Mean to Better Health}

Health and healthcare is undergoing remarkable changes. Over the next few years healthcare will flip almost 180 degrees. First, the emphases on health and behavior modification will keep most people out of the hospital. Technology and the tools produced will become strong motivators for healthy lifestyles including nutrition, exercise, and better behavioral habits. The increased use of mobile devices, wearable sensors, and patient reported data shift the focus of healthcare outside of the hospital. A consequence of this pattern shift will mean the people who use the current system will have to pay more for illness care. More importantly, large institutions will have to scale down both in size and staffing. Healthcare will shift from brick and mortar to care where the patient is.

The use of machine learning and artificial intelligence used with Big Data will create new knowledge that will rapidly be applied to patient care. With the recognition of new data types and the integration of that data into the EHR and into decision making, humans will be outclassed, and computers will become the decision makers in healthcare, and the role of clinicians will change. Rich sets of phenotypes and care plans - whether inpatient or home-based will become the norm for applying knowledge to care. Parallel processers and cloud computing will accommodate the demands for faster computing. With every input of new data, a person's health condition will be completely reevaluated. This reevaluation may trigger a thousand decision support algorithms. Ultimately there may be a processor chip per person. Many of the tasks currently carried out will be performed by robots. Virtual and augmented reality will be used for viewing data, for education, and for entertainment. All of these changes will have a professional impact, an economic impact, and a social impact.

This new world will be dependent on the liquidity of data. Interoperability becomes even more critical. Transport standards, a universal common set of complete data elements, open source APIs, phenotype development, new workflows, new data structures, and new data architectures will be only a few of the new requirements facing the technical community.

One of the challenges that must be overcome is how can we keep our systems up to date with new technology, new policies, new models and new demands. Most of the current commercial EHR systems, for example, are based on a database architecture that is over 40 years old. With large customer bases and billions of invested dollars in current systems, how can we accommodate change? A suggestion is to move all functionality from the EHR system itself. The EHR should become simply a container to input and output data (a push and pull operation) supporting a global common data element database. All functions supporting the capture of data, the analysis of data, and the presentation of data will be external to the EHR. These functions can evolve with new technologies and new requirements can be more quickly created and built into work flows.

\section{The World as it is}

Today's world is largely siloed. We tend to deal with new problems or requirements one at a time. We live with many less than ideal solutions that may only partially solve the problem. We do not have an effective way of bringing all the interested parties together in a comprehensive way to mutually find tomorrow's solution. The section below discusses some case examples of this issue.

Interoperability begins with being able to uniquely identify a person from any and every source. The only logical solution to this problem is a Universal Person Identifier (UPI). The U.S. is the last developed country that does not have a UPI. We attempt to identify a person with between 12 15 different data elements that may or may not be correct or present. The consequence is an error rate that exceeds $10 \%$. That error rate prevents the level of accuracy to aggregate data across multiple sites. For example, one cannot create a medications list across multiple institutions with sufficient accuracy to make the list reliable and hence usable.

The second problem that must be solved is a common language across all healthcare settings and inclusive of all 
clinical domains. The current standard sets of preferred controlled terminologies is inadequate to support an information loss-free exchange and aggregation of data. There are too many choices to represent a concept consistently within a single institution much less across an enterprise, region, country, or the global community. The set of attributes such as units, value sets, data types and other characteristics are not standardized. The granularity of data items is not defined at the atomic level required to drive many decision support algorithms. The problem is compounded by the fact that many different groups are attempting to define a common data element set that differs from other groups.

Most of controlled terminologies deal only with a specific classification of terminologies such as diagnoses, procedures, laboratory tests, or medications. Administrative terms are frequently not defined. For example, what is an inpatient? Does an ambulatory visit in which the patient is kept overnight become an inpatient visit? There is no consistent answer to this question. What is the time of discharge of a patient? Is it when the discharge order is written? Is it when the patient leaves the hospital room? Is it when the patient checks out administratively? Analytics require specific definitions of these and similar terms.

A common method for transporting data to other locations is in the form of a document or container. HL7's Clinical Document Architecture (CDA) [9] has been used to define a family of the Continuity of Care Document (CCD) based on the CDA as an electronic document exchange standard for sharing patient summary information. Another Standards Developing Organization (SDO) - Integrating the Health Enterprise (IHE) - has developed an Implementation Guide called Structured Document Capture (SDC) [10] based on the Retrieve Form for Data Capture Profile (RFD). RFD supports the retrieval of a form from a form source, the display and completion of the form, and the return of instance data from the display application to a receiving application. The European for Standardization (CEN) standard, CEN/ISO EN13606 [11], transports data in folders. Most systems store these documents as part of the EHR making it more difficult to extract dynamically the level of granularity required for analytics and decision support. Most data retrieval tools do not support the extraction of atomic data elements from these stored documents.

Specific data elements are generally stored within the contents of the lab or diagnostic test or source of the data. In many cases, a specific data element might be a component of several different tests but is, in fact, the same data element. If the data is stored only within the larger component, a retrieval program must have the knowledge to look at all possible sources to see if the test exists. Finding that a specific data element has never been collected is often a challenging and time consuming task. A new approach to database architecture needs to be approached. Rather than a relational database, we need to consider a noSQL [12] approach in which the storage architecture needs to be driven by operational requirements rather than a table structure. Epic uses two types of databases in that a Mumps/M database is used for a single patient's record and a standard relational database (Clarity) is use when searching across patients.

If EHR data is to be used for pragmatic clinical trials, then that data needs to have the highest quality possible. Data must be precise, complete, accurate and consistent. To whatever extent possible, data quality must be checked as part of the data capture. For example, males cannot be pregnant; women do not have prostate cancer. Simple rules applied at the time of data capture can be applied. These rules may be gender or conditional specific. They may be driven by limits. They may be checked by algorithms such as delta-check. The same approach works for consistency.

\section{Evolving Standards}

HL7 has been developing standards for over 30 years. The first HL7 standard, v2, was developed specifically to enable the creation of a Hospital Information System (HIS) using a Best of Breed approach. The competition was a single integrated system that did everything. Data needed to be passed among the various components of an HIS. The requirements were well defined, and the v2 standard evolved around the concept of segments based on classification of data - demographic patient data, test ordering and result reporting, encounter data, financial data, etc. The $\mathrm{Z}$ segment was added to permit implementers to add any new data element not specifically defined within the standard. Version 2 standards are still in wide use today, especially in the U.S. It is not however an interoperable standard.

Version 3 was based on a Reference Information Model and was more complex and challenging to understand and implement. The CDA resulted from the integration of another group into HL7 and had excellent international implementation. The combining of an ASTM effort, the Continuity of Care Record (CCR) into the CDA standard to create the CCD was a substantial step forward toward interoperability. One of the frustrations to the CCD was that you had to send everything defined within the CCD and there was no freedom to add additional data elements.

The user community pushed back strongly against v3, and there were data exchange needs not met by CDA. At an HL7 Board Retreat, the idea was voiced to create of a Fresh Look Task Force to investigate looking to develop a new standard based on the experience of the past 25 years of creating standards. A task force was created, and in July 2011, Graham Grieve came up with the concept on a new standard named FHIR ${ }^{\circledR}$ (Fast Healthcare Interoperability Resources ${ }^{\circledR}$ ). FHIR captures the simplicity and flexibility of v2 and is rapidly becoming the standard for interoperability. FHIR is based on a set of modules called Resources. Resources are common basic building blocks by which all data exchanges occur. Resources permit the transporting of data at the lowest levels of granularity or at any level of packaged data. Examples of Resources are Patient, Practitioner, Allergy, Family History, and Care Plan. FHIR uses a web services technology (RESTful APIs) to exchange Resources between groups. FHIR is a service-driven application. Resources 
are combined in Profiles to support clinical, administrative, or other functionalities. Profiles also may constrain the content of Resources, so only the data required by the service is exchanged. Regardless of the paradigm, the content is the same.

Clearly most of the changes that are taking place depend on interoperable data sharing, enabled by standards, a common set of data elements, and high data quality. The Senate and Congress of the U.S. has passed the $21^{\text {st }}$ Century Cures Act [13] that defines a trusted interoperable framework and common agreement for EHR transparency, usability, security, and functionality. This law requires EHR vendors to make patient data accessible without special effort, through the use of application programming interfaces. These APIs will be a common technology used by everyone to exchange data bidirectionally with EHRs, independent of the vendor. In other words, the APIs support both push data into the EHR or to pull/query data from any EHR. A team at Harvard has defined a set of rules for substitutable medical apps $\&$ reusable technology (SMART) [14]. The combination of these specifications along with FHIR (called SMART on FHIR) appears to be the tool of choice for meeting the requirements of the $21^{\text {st }}$ Century Cures Act.

\section{Better Health}

Better health means getting the right data to the right place to the right person who uses the right knowledge to make the right diagnosis and starts the right treatment. No diagnosis or the wrong diagnosis is the norm for the present system. Only $4 \%$ of people with rare disease are correctly diagnosed. The reason is frequently a lack of access to a complete set of quality data. Clinicians have trouble finding the data they need in a timely fashion. Medication errors are the most common type of medical error. These errors are a result of the wrong dosage, interactions among drugs, incorrect medication, illegible handwriting of prescriptions, and allergies unknown or missed.

What is the role of HL7 International in making sure that we help build the New World to ensure Better Health? When HL7 first started, it had a focused view about what was required. Over time, that view began to spread as new requirements were identified and new groups came into the organization. HL7 reached out to the international community, to other SDOs and technical groups (leading to CDA), to the clinical decision support community, to the clinical community, to governments, and now to the genomic community. The changes in health, health care, and technology will lead to new requirements. HL7 needs to be out front in defining what is needed, and making sure someone is accepting responsibility.

It is my belief that computers and AI will become much more dominant in bringing Better Health forward. Computers have the ability to consistently follow a set of rules. Humans frequently miss those opportunities. An example to illustrate that point is the case of harvesting donor organs. Duke, unfortunately, harvests only about 1 in 4 of available donor organs. If a computer program could be triggered by data that suggested an organ might become available for harvest and notify appropriate persons, then track that process throughout the encounter, that number should become 4 out of 4 . Having processes start as a result of certain data entry, i.e., a trigger to manage data flow or work flow would make a significant contribution to better health. CDS Hooks is a step in this direction.

\section{References}

[1] Blobel B. Translational Medicine Meets New Technologies for Enabling Personalized Care. Stud Health Technol Inform. 2013; 189: 8-23.

[2] Ortman JM, Velkoff VA, Hogan H. An Aging Nation: The Older Population in the United States. U.S. Census Bureau, 2014.

[3] Blobel B. Paradigm Changes of Health Systems Towards Ubiquitous, Personalized Health Leads to Paradigm Changes of Security and Privacy Ecosystems. Int J Biomedicine and Healthcare. 2015; 3: 6-11.

[4] McGinnis JM, Williams-Russo P, Knickman JR. The Case for More Active Policy Attention to Health Promotion. Health Affairs. 2012; 21: 78-93.

[5] www.nihcollaboratory.gov

[6] www.pcornet.org

[7] www.apple.com

[8] https://www.census.gov/prod/2014pubs/p25-1140.pdf

[9] www.hl7.org

[10] www.healthit.gov/policy-researchers-implementers/structured-datacapture-sdc.

[11] Martínez-Costa C, Menárguez-Tortosa M, Fernández-Breis TJ. An approach for the semantic interoperability of ISO EN 13606 and OpenEHR archetypes. J. Biomed. Inform. 2010; 43: 736-746.

[12] Sánchez-de-Madariaga R, Muñoz A, Lozano-Rubí R, Serrano-Balazote P, Castro AL, Moreno O, Pascual M. Examining database persistence of ISO/EN 13606 standardized electronic health record extracts: relational vs. NoSQL approaches. BMC Med Inform Decis Mak. 2017; 17: 123.

[13] www.congress.gov/bill/114th-congress/house-bill/6

[14] https://smarthealthit.org/ 